\title{
Precise Control of a Four Degree-of-Freedom Permanent Magnet Biased Active Magnetic Bearing System in a Magnetically Suspended Direct-Driven Spindle using Neural Network Inverse Scheme
}

\author{
Xiaodong Sun ${ }^{1,2}$, Bokai $\mathrm{Su}^{1}$, Long Chen ${ }^{1,2}$, Zebin Yang ${ }^{3}$, Xing $\mathrm{Xu}^{1,2}$, and Zhou Shi ${ }^{1}$ \\ 1. School of Automobile and Traffic Engineering, Jiangsu University, Zhenjiang 212013, Jiangsu, China; \\ 2. Automotive Engineering Research Institute, Jiangsu University, Zhenjiang 212013, Jiangsu, China \\ 3. School of Electrical and Information Engineering, Jiangsu University, Zhenjiang 212013, Jiangsu, China \\ *Corresponding author \\ Telephone/Fax: +86-0511-88782845; Email: xdsun@ujs.edu.cn
}

\begin{abstract}
The capacity of improving the control accuracy and dynamic performance of a four degreeof-freedom (DOF) permanent magnet biased active magnetic bearing (PMBAMB) system is critical to developing and maintaining a high precision application in a magnetically suspended direct-driven spindle system. The 4-DOF PMBAMB system, however, is a multivariable, strong coupled and nonlinear system with unavoidable and unmeasured external disturbances, in addition to having parameter variations. The satisfactory control performance cannot be obtained by using traditional strategies. Therefore, it is important to present a novel control scheme to construct a robust controller with good closed-loop capability. This paper proposes a new decoupling control scheme for a 4-DOF PMBAMB in a direct-driven spindle system based on the neural network inverse (NNI) and 2- degreeof-freedom (DOF) internal model control method. By combining the inversion of the 4-DOF PMBAMB system with its original system, a new pseudolinear system can be developed. In addition, by introducing the 2-DOF internal model controller into the pseudolinear system to design extra closed-loop controllers, we can effectively eliminate the influence of the unmodeled dynamics to the decoupling control accuracy, as well as adjust the properties of tracking and disturbance rejection independently. The experimental results demonstrate the effectiveness of the proposed control scheme.
\end{abstract}

Keywords: Magnetic bearing; neural network inverse; decoupling control; internal model control; magnetically suspended direct-driven spindle.

\section{Introduction}

Compared with conventional mechanical bearings, magnetic bearings (MBs) possess several remarkable 
advantages, such as no friction and wear, no need of lubrication, long life span, high potential of high control precision, as well as the ability of long-term high speed running [1]. Therefore, MBs have been attracting considerable interests in various high-performance applications including flywheel energy and storage devices, bearingless motors, artificial heart pumps, vacuum pumps, numerical control machines, especially in special high-purity applications (e.g., pharmaceutical mixing, bioreactor mixing, etc.). Furthermore, in some special environments, such as at temperature extremes and in vacuum operations, MBs have the irreplaceable opportunity due to their obvious non-contact and frictionless characteristics $[2 ; 3 ; 4]$.

Owing to the utilization of rare earth permanent magnet, permanent magnetic biased hybrid magnetic bearings (PMBAMBs) have characteristics of high efficiency, small size, and low running cost [5]. For these advantages, PMBAMBs have been widely adopted to take the place of the extra currents to produce the radial suspension forces. However, to design an available control scheme for a PMBAMB is still a challenging topic especially in a multi-DOF PMBAMB system due to its highly nonlinear, strong coupled, and open-loop unstable control characteristics. Thus, a sophisticated control scheme which is capable of regulating and stabilising the rotor of the PMBAMB system within a narrow airgap is of the essence in different operating environments [6].

As a traditional control scheme, the proportional plus integral plus differential (PID) control scheme has been already applied in the MB system in a general way owing to its simple realization [7; 8]. However, the control performance of the MB system by using the conventional PID control scheme could not be very satisfied owe to the unmeasured parameters variations and unavoidable external disturbances. Therefore, with the development of modern control theories, all kinds of advanced control methods have been recently proposed for MB systems, such as fuzzy control [9], intelligent control [10], sliding mode control [11], predictive control [12], robust control [13], adaptive control [14; 15], fractional order control [16], backstepping control [17], optimum control [18], etc. These advanced control methods not only enrich the control theory of MB systems, but also improve their performance in different aspects. The fuzzy control scheme can express a nonlinear magnetic levitation drive system by the time-varying convex combination of linear state space models utilizing nonlinear fuzzy membership functions so that it is possible to use other control technique. The sliding mode control scheme can guarantee asymptotic and finite-time tracking capabilities of the magnetic levitation drive system. The predictive control scheme can decrease the settling time remarkably and reduce oscillations in the lower part of operating range effectively. The adaptive control scheme can achieve simultaneous control of both rotor vibrations and transmitted forces in flexible magnetic suspension rotor systems. The backstepping control scheme uses the nonlinear observers to provide the estimate of the unmeasured state and can achieve high-quality control of the magnetic levitation drive system in the case of 
acceleration disturbances at different rotational speeds. The fractional order control scheme employs a new error criterion by combining the mean square error with a maximal phase error to find the optimal model with smaller phase errors.

Besides aforementioned nonlinear control methods, the linearization and decoupling control schemes have been widely employed for MB systems in recent years [19]. In general, the linearization and decoupling scheme can be divided into the differential geometry decoupling scheme and the inverse system scheme $[20 ; 21]$. Since the differential geometry scheme is abstractive and needs to convert the control problem to the geometric domain, it is difficult to be popularized in practical applications. Additionally, the inverse system scheme is simple, intuitive, and relatively simple to carry out. In [22], the inverse system scheme is used for the radial position control of a magnetically suspended rotor system in a direct-driven spindle. However, attributed to the complexity of the magnetically suspended rotor system in a direct-driven spindle, its mathematical model will not be known precisely. So the motivation of linearization and decoupling is hard to achieve by only utilizing the inverse system scheme. The other approach is needed to combine with the inverse system method to realize the linearization and decoupling control.

On the other hand, the neural network is one of the intelligent control methods which does not need mathematical model of the controlled plant and can effectively approximate nonlinear systems [23; 24; 25]. With the well-developed on-line learning and self-adapting abilities, the neural networks are capable of dealing with highly nonlinear and time-varying systems with the unmeasured parameters variations, as well as the unavoidable external disturbances. Therefore, by using the neural network to construct inverse system, a new control method, the so called neural network inverse (NNI), is proposed [26].

Since the developed pseudo-linear system of 4-DOF PMBAMB system by using the NNI control scheme is not a simple linear system, the parameters variations may inevitably influence the properties of decoupling, tracking, and disturbance rejection performance. The traditional internal model control can obtain good performance for set point tracking, but gives the sluggish response for disturbance rejection problem. Thus, it is difficult for traditional internal model control to give consideration to both tracking and disturbance rejection properties. Therefore, for purpose of improving the static and dynamic properties of the whole 4-DOF PMBAMB system, and adjusting the tracking and disturbance rejection performances independently, the NNI control scheme plus 2- DOF internal model controllers are adopted in the paper.

The paper is organized as follows. First, in Section 2, we construct the model of the 4-DOF PMBAMB system in a magnetically suspended direct-driven spindle system and analyze its dynamic characteristics. After that, the NNI control scheme is adopted for decoupling control of the 4-DOF PMBAMB system in Section 3. Second, the internal model control theory is used to design the closed- 
loop controllers to improve system robustness in Section 4. Then, the comparative experimental researches between the proposed method and the traditional PID control scheme are carried out in Section 5. Finally, Section 6 concludes this paper.

\section{4-DOF PMBAMB System in a Magnetically Suspended Direct-Driven Spindle System}

\subsection{System Structure}

The magnetically suspended direct-driven spindle system has 6-DOFs, i.e., a rotational DOF and 5 suspended DOFs. The fully suspended 5-DOF PMBAMB system in the magnetically suspended directdriven spindle system includes four radial DOFs controlled by two 2-DOF radial PMBAMBs and one axial DOF controlled by a single-DOF axial MB. Since there is no coupling between the single-DOF axial MB and the 4-DOF radial PMBAMBs, we mainly focus on the nonlinear decoupling control of the 4-DOF PMBAMB system in this paper (the coupling characteristics of the 4-DOF PMBAMB will be analyzed in Section 2.3).

Fig. 1 illustrates the structure of the adopted magnetically suspended direct-driven spindle system. A single-DOF axial MB and two 2-DOF radial PMBAMBs are used to suspend and regulate the rotor in the axial and radial DOFs, respectively. Two pairs of the perpendicular eddy-current displacement sensors (i.e. gap sensors) are installed closely to respective 2-DOF radial PMBAMBs to measure the respective radial displacements in perpendicular directions. Additionally, an eddy-current displacement sensor is installed closely to the single-DOF axial MB to measure the axial displacement in axial direction. After the rotor displacements in five directions are all measured and sent to the control unit through the signal lines and the interface circuit, the required electromagnet currents can be generated to regulate and stabilize the rotor in the airgap center of two 2-DOF radial PMBAMBs.

\section{Fig. 1 HERE}

\subsection{System Modeling}

In this paper, the rotor of the magnetically suspended direct-driven spindle system is assumed to be a rigid and symmetric body. Fig. 2 shows the relationship of the coordinate system and the rotor forces of the 4-DOF PMBAMBs. In Fig. 2, $O$ is the geometrical center of the rotor; $O_{A}$ and $O_{B}$ denote the two centers of the two 2-DOF radial PMBAMBs, respectively; $X-, Y$-, and $Z$-axes form the coordinate system of the rotor position; $x$ and $y$ are the radial displacements in $X$ - and $Y$-axes, respectively; $\theta_{x}$ and $\theta_{y}$ are the rotor angular displacements about the $X$ - and $Y$-axes, respectively; $\left[x, \theta_{y}, y, \theta_{x}\right]^{\mathrm{T}}$ form the generalized coordinates of the rotor system; $F_{a x}, F_{b x}, F_{a y}$, and $F_{b y}$ are the radial AMB forces along the $A X-, B X-, A Y-$, and $B Y$ - axes, respectively; $\Omega$ is the rotational mechanical speed of the rotor.

Fig. 2 HERE 
By Newton's second law, the dynamic model of 4-DOF PMBAMB system can be obtained according to its structure as follows:

$$
\left\{\begin{array}{l}
m \ddot{x}=F_{a x}+F_{b x} \\
m \ddot{y}=F_{a y}+F_{b y}-m g \\
J_{x} \ddot{\theta}_{x}=F_{b y} l_{b}-F_{a y} l_{a}-J_{z} \Omega \dot{\theta}_{y} \\
J_{y} \ddot{\theta}_{y}=F_{a x} l_{a}-F_{b x} l_{b}+J_{z} \Omega \dot{\theta}_{x}
\end{array}\right.
$$

where $m$ is the mass of the rotor; $g$ is the gravity constant; $x$ and $y$ are the radial displacements of the mass center of the rotor from $O$ in the X-and Y-axes, respectively; $l_{a}$ and $l_{b}$ are the distance from $O$ to the centers of the two 2-DOF radial PMBAMBs, respectively; $J_{x}, J_{y}$, and $J_{z}$ are the moments of inertia of the rotor in the $X-, Y$-, and $Z$-axes, respectively, and $J_{x}=J_{y}$.

Neglecting the influence of magnetic saturation and leakage, the radial AMB forces of the 4-DOF PMBAMB system can be expressed as follows

$$
\left\{\begin{array}{l}
F_{a x}=K_{1} x_{a}+K_{2} i_{a x} \\
F_{a y}=K_{1} y_{a}+K_{2} i_{a y} \\
F_{b x}=K_{1} x_{b}+K_{2} i_{b x} \\
F_{b y}=K_{1} y_{b}+K_{2} i_{b y}
\end{array}\right.
$$

where $x_{a}, x_{b}, y_{a}$, and $y_{b}$ are the radial displacements of the rotor in the $A X_{-}^{-}, B X_{-}^{-}, A Y_{-}$, and $B Y$-axes, respectively, and $i_{a x}, i_{b x}, i_{a y}$, and $i_{b y}$ are the control current components in the $A X-, B X-, A Y$-, and $B Y$-axes, respectively. And $\left[x_{a}, x_{b}, y_{a}, y_{b}\right]^{\mathrm{T}}$ form the coordinates of the 2-DOF radial PMBAMB. $K_{1}$ and $K_{2}$ are the displacement and current stiffness coefficients, respectively, and can be defined as follows:

$$
\left\{\begin{array}{l}
K_{1}=3 \mu_{0} F_{m}^{2} S_{m} / 4 \delta_{0}^{3} \\
K_{2}=3 \mu_{0} N F_{m} S_{m} / 2 \delta_{0}^{2}
\end{array}\right.
$$

where $\delta_{0}$ is the nominal radial airgap length, $\mu_{0}$ is the magnetic permeability of the air, $F_{m}$ is the magnetomotive force provided by the permanent magnet, $S_{m}$ is the pole face area, $N$ is the number of coil turns.

\subsection{Analysis of Coupling Characteristics}

From Fig. 2, the coordinate conversion from the coordinates of the 2-DOF radial PMBAMB to the generalized coordinates of the rotor system can be expressed as follows:

$$
\left\{\begin{array}{l}
x=l_{b} x_{a} /\left(l_{a}+l_{b}\right)+l_{a} x_{b} /\left(l_{a}+l_{b}\right) \\
y=l_{b} y_{a} /\left(l_{a}+l_{b}\right)+l_{a} y_{b} /\left(l_{a}+l_{b}\right) \\
\theta_{x}=\left(y_{b}-y_{a}\right) /\left(l_{a}+l_{b}\right) \\
\theta_{y}=\left(x_{a}-x_{b}\right) /\left(l_{a}+l_{b}\right)
\end{array}\right.
$$

Bringing (4) into (1), we can get 


$$
\left\{\begin{array}{l}
F_{a x}+F_{b x}=\frac{m}{l_{a}+l_{b}}\left(l_{b} \ddot{x}_{a}+l_{a} \ddot{x}_{b}\right) \\
F_{a y}+F_{b y}-m g=\frac{m}{l_{a}+l_{b}}\left(l_{b} \ddot{y}_{a}+l_{a} \ddot{y}_{b}\right) \\
F_{b y} l_{b}-F_{a y} l_{a}-\frac{J_{z} \Omega}{l_{a}+l_{b}}\left(\dot{x}_{a}-\dot{x}_{b}\right)=\frac{J_{x}}{l_{a}+l_{b}}\left(\ddot{y}_{b}-\ddot{y}_{a}\right) \\
F_{a x} l_{a}-F_{b x} l_{b}+\frac{J_{z} \Omega}{l_{a}+l_{b}}\left(\dot{y}_{b}-\dot{y}_{a}\right)=\frac{J_{y}}{l_{a}+l_{b}}\left(\ddot{x}_{a}-\ddot{x}_{b}\right)
\end{array}\right.
$$

Substituting the first expression into the fourth expression of (5) gives

$$
F_{a x}=\frac{m l_{b}}{\left(l_{a}+l_{b}\right)^{2}}\left(l_{b} \ddot{x}_{a}+l_{a} \ddot{x}_{b}\right)+\frac{J_{y}}{\left(l_{a}+l_{b}\right)^{2}}\left(\ddot{x}_{a}-\ddot{x}_{b}\right)-\frac{J_{z} \Omega}{\left(l_{a}+l_{b}\right)^{2}}\left(\dot{y}_{b}-\dot{y}_{a}\right)
$$

In the case of $\Omega=0$, (5) can be rewritten as

$$
F_{a x}=\frac{m l_{b}}{\left(l_{a}+l_{b}\right)^{2}}\left(l_{b} \ddot{x}_{a}+l_{a} \ddot{x}_{b}\right)+\frac{J_{y}}{\left(l_{a}+l_{b}\right)^{2}}\left(\ddot{x}_{a}-\ddot{x}_{b}\right)
$$

Taking the $A X$ - axis radial channel as an example, from (7), it is obvious that $F_{a x}$ is the function of $\ddot{x}_{a}$ and $\ddot{x}_{b}$. Therefore, in this case, $A X$ - axis radial channel is only dynamically coupled with $B X$ - axis radial channel. We can draw the similar conclusions for the other three radial channels.

Similar conclusions can be drawn for the other three channels.

Under the condition $\Omega \neq 0$, however, it is clear that $F_{a x}$ is the function of $\ddot{x}_{a}, \ddot{x}_{b}, \dot{y}_{a}$ and $\dot{y}_{b}$. That is, $A X$ - axis radial channel is dynamically coupled with $B X-, A Y$-, and $B Y$ - axes radial channels. Also, the similar conclusions can be drawn for the other three radial channels. Therefore, in the case of $\Omega \neq 0$, the dynamic couplings always exist among the four radial channels.

From the above analysis, the 4-DOF PMBAMB system is represented to be multivariable strongly coupled.

\section{Decoupling Control of the 4-DOF PMBAMB System Using NNI Scheme}

Since the purpose of control is to decouple the radial displacements $x_{a}, x_{b}, y_{a}$, and $y_{b}$, the output variables are $\boldsymbol{Y}=\left[y_{1}, y_{2}, y_{3}, y_{4}\right]^{T}=\left[x_{a}, x_{b}, y_{a}, y_{b}\right]^{T}$. Moreover, choose $i_{a x}, i_{b x}, i_{a y}$, and $i_{b y}$ to be the control variables, then $\boldsymbol{U}=\left[u_{1}, u_{2}, u_{3}, u_{4}\right]^{T}=\left[i_{a x}, i_{b x}, i_{a y}, i_{b y}\right]^{T}$. Choose $x, \theta_{y}, y, \theta_{x}$ and their first-order derivatives to be state variables, then $\boldsymbol{X}=\left[x_{1}, x_{2}, x_{3}, x_{4}, x_{5}, x_{6}, x_{7}, x_{8}\right]^{T}=\left[x, \theta_{y}, y, \theta_{x}, \dot{x}, \dot{\theta}_{y}, \dot{y}, \dot{\theta}_{x}\right]^{T}$. Consequently, the corresponding state equation of the nonlinear system (1) can be rewritten as

$$
\left\{\begin{array}{l}
\dot{\boldsymbol{X}}=f(\boldsymbol{X}, \boldsymbol{U}) \\
\boldsymbol{Y}=\boldsymbol{C} \boldsymbol{X}
\end{array}\right.
$$


where $f(\boldsymbol{X}, \boldsymbol{U})$ and matrix $\boldsymbol{C}$ can be expressed in (9) and (10).

$$
\begin{aligned}
& f(\boldsymbol{X}, \boldsymbol{U})= \\
& {\left[\begin{array}{l}
x_{5} \\
x_{6} \\
x_{7} \\
x_{8} \\
\frac{1}{m}\left\{K_{1}\left[2 x_{1}+x_{2}\left(l_{b}-l_{a}\right)\right]+K_{2}\left(u_{1}+u_{2}\right)\right\} \\
\frac{1}{J_{y}}\left\{l_{a}\left[K_{1}\left(x_{1}-l_{a} x_{2}\right)+K_{2} u_{1}\right]-l_{b}\left[K_{1}\left(x_{1}+l_{b} x_{2}\right)+K_{2} u_{2}\right]+J_{z} \Omega x_{8}\right\} \\
\frac{1}{m}\left\{K_{1}\left[2 x_{3}+x_{4}\left(l_{b}-l_{a}\right)\right]+K_{2}\left(u_{3}+u_{4}\right)\right\}-g \\
\frac{1}{J_{x}}\left\{l_{b}\left[K_{1}\left(x_{3}+l_{b} x_{4}\right)+K_{2} u_{4}\right]-l_{a}\left[K_{1}\left(x_{3}-l_{a} x_{4}\right)+K_{2} u_{3}\right]+J_{z} \Omega x_{6}\right\}
\end{array}\right]} \\
& \boldsymbol{C}=\left[\begin{array}{llllllll}
1 & -l_{a} & 0 & 0 & 0 & 0 & 0 & 0 \\
1 & l_{b} & 0 & 0 & 0 & 0 & 0 & 0 \\
0 & 0 & 1 & -l_{a} & 0 & 0 & 0 & 0 \\
0 & 0 & 1 & l_{b} & 0 & 0 & 0 & 0
\end{array}\right]
\end{aligned}
$$

According to the inverse system theory and Interactor algorithm, the outputs $\boldsymbol{Y}=\left[y_{1}, y_{2}, y_{3}, y_{4}\right]^{\mathrm{T}}$ of the system are differentiated with respect to time until the derivatives contain input $\boldsymbol{U}=\left[u_{1}, u_{2}, u_{3}, u_{4}\right]^{\mathrm{T}}$ obviously. Then, we can obtain (11).

$$
\begin{aligned}
& \boldsymbol{J}(\boldsymbol{U})=\left[\begin{array}{llll}
\ddot{y}_{1} & \ddot{y}_{2} & \ddot{y}_{3} & \ddot{y}_{4}
\end{array}\right]^{T} \\
& {\left[\frac{1}{m}\left\{K_{1}\left[2 x_{1}+x_{2}\left(l_{b}-l_{a}\right)\right]+K_{2}\left(u_{1}+u_{2}\right)\right\}-\frac{l_{a}}{J_{y}}\left\{l_{a}\left[K_{1}\left(x_{1}-l_{a} x_{2}\right)+K_{2} u_{1}\right]-l_{b}\left[K_{1}\left(x_{1}+l_{b} x_{2}\right)+K_{2} u_{2}\right]+J_{z} \Omega x_{8}\right\}\right.} \\
& =\frac{1}{m}\left\{K_{1}\left[2 x_{1}+x_{2}\left(l_{b}-l_{a}\right)\right]+K_{2}\left(u_{1}+u_{2}\right)\right\}+\frac{l_{b}}{J_{y}}\left\{l_{a}\left[K_{1}\left(x_{1}-l_{a} x_{2}\right)+K_{2} u_{1}\right]-l_{b}\left[K_{1}\left(x_{1}+l_{b} x_{2}\right)+K_{2} u_{2}\right]+J_{z} \Omega x_{8}\right\} \\
& \frac{1}{m}\left\{K_{1}\left[2 x_{3}+x_{4}\left(l_{b}-l_{a}\right)\right]+K_{2}\left(u_{3}+u_{4}\right)\right\}-g-\frac{l_{a}}{J_{x}}\left\{l_{b}\left[K_{1}\left(x_{3}+l_{b} x_{4}\right)+K_{2} u_{4}\right]-l_{a}\left[K_{1}\left(x_{3}-l_{a} x_{4}\right)+K_{2} u_{3}\right]+J_{z} \Omega x_{6}\right\} \\
& \left.\frac{1}{m}\left\{K_{1}\left[2 x_{3}+x_{4}\left(l_{b}-l_{a}\right)\right]+K_{2}\left(u_{3}+u_{4}\right)\right\}-g+\frac{l_{b}}{J_{x}}\left\{l_{b}\left[K_{1}\left(x_{3}+l_{b} x_{4}\right)+K_{2} u_{4}\right]-l_{a}\left[K_{1}\left(x_{3}-l_{a} x_{4}\right)+K_{2} u_{3}\right]+J_{z} \Omega x_{6}\right\}\right]
\end{aligned}
$$

Thus, the Jacobi matrix $\boldsymbol{D}$ can be achieved as follows:

$$
\boldsymbol{D}=\frac{\partial \boldsymbol{J}}{\partial \boldsymbol{U}}=\left[\begin{array}{llll}
\frac{\partial \ddot{y}_{1}}{\partial u_{1}} & \frac{\partial \ddot{y}_{1}}{\partial u_{2}} & \frac{\partial \ddot{y}_{1}}{\partial u_{3}} & \frac{\partial \ddot{y}_{1}}{\partial u_{4}} \\
\frac{\partial \ddot{y}_{2}}{\partial u_{1}} & \frac{\partial \ddot{y}_{2}}{\partial u_{2}} & \frac{\partial \ddot{y}_{2}}{\partial u_{3}} & \frac{\partial \ddot{y}_{2}}{\partial u_{4}} \\
\frac{\partial \ddot{y}_{3}}{\partial u_{1}} & \frac{\partial \ddot{y}_{3}}{\partial u_{2}} & \frac{\partial \ddot{y}_{3}}{\partial u_{3}} & \frac{\partial \ddot{y}_{3}}{\partial u_{4}} \\
\frac{\partial \ddot{y}_{4}}{\partial u_{1}} & \frac{\partial \ddot{y}_{4}}{\partial u_{2}} & \frac{\partial \ddot{y}_{4}}{\partial u_{3}} & \frac{\partial \ddot{y}_{4}}{\partial u_{4}}
\end{array}\right]=\left[\begin{array}{cccl}
\frac{1}{m} k_{2}-\frac{l_{a}^{2}}{j_{y}} k_{2} & \frac{1}{m} k_{2}+\frac{l_{a} l_{b}}{j_{y}} k_{2} & 0 & 0 \\
\frac{1}{m} k_{2}+\frac{l_{a} l_{b}}{j_{y}} k_{2} & \frac{1}{m} k_{2}-\frac{l_{b}^{2}}{j_{y}} k_{2} & 0 & 0 \\
0 & 0 & \frac{1}{m} k_{2}+\frac{l_{a}^{2}}{j_{x}} k_{2} & \frac{1}{m} k_{2}-\frac{l_{a} l_{b}}{j_{x}} k_{2} \\
0 & 0 & \frac{1}{m} k_{2}-\frac{l_{a} l_{b}}{j_{x}} k_{2} & \frac{1}{m} k_{2}+\frac{l_{b}^{2}}{j_{x}} k_{2}
\end{array}\right]
$$

Hence, $\operatorname{det}(\boldsymbol{D})$ can be obtained as 


$$
\operatorname{det}(\boldsymbol{D})=K_{2}^{4}\left[\left(\frac{1}{m}-\frac{l_{a}^{2}}{J_{y}}\right)\left(\frac{1}{m}-\frac{l_{b}^{2}}{J_{y}}\right)-\left(\frac{1}{m}+\frac{l_{a} l_{b}}{J_{y}}\right)\left(\frac{1}{m}+\frac{l_{a} l_{b}}{J_{y}}\right)\right]\left[\left(\frac{1}{m}+\frac{l_{a}^{2}}{J_{x}}\right)\left(\frac{1}{m}+\frac{l_{b}^{2}}{J_{x}}\right)-\left(\frac{1}{m}-\frac{l_{a} l_{b}}{J_{x}}\right)\left(\frac{1}{m}-\frac{l_{a} l_{b}}{J_{x}}\right)\right]
$$

Obviously, $K_{2}$ will not be zero. Thus, $\operatorname{det}(\boldsymbol{D})=0$ if and only if at least one of the two product factors equals to zero. Supposing the first one equals to zero, i.e.,

$$
\left(\frac{1}{m}-\frac{l_{a}^{2}}{J_{y}}\right)\left(\frac{1}{m}-\frac{l_{b}^{2}}{J_{y}}\right)=\left(\frac{1}{m}+\frac{l_{a} l_{b}}{J_{y}}\right)\left(\frac{1}{m}+\frac{l_{a} l_{b}}{J_{y}}\right)
$$

By solving (14), we have

$$
l_{a}+l_{b}=0
$$

Similarly, the same conclusion can be drawn when the other holds. Hence, $\operatorname{det}(\boldsymbol{D})=0$ if and only if $l_{a}=-l_{b}$. Note that the values of $l_{a}$ and $l_{b}$ are greater than zero in practice. Therefore, the inequality $\operatorname{det}(\boldsymbol{D})$ $\neq 0$ always satisfies in the actual control system. Therefore, the relative order of the system is $\alpha=\left(\alpha_{1}, \alpha_{2}\right.$, $\left.\alpha_{3}, \alpha_{4}\right)=(2,2,2,2)$, which satisfies $\alpha_{1}+\alpha_{2}+\alpha_{3}+\alpha_{4}=8 \leqslant n,(n$ is the number of the state variables) [27]. Thus, in accordance with the inverse system theory, we can conclude that the inverse of the original system is existent. And the inverse system can be written as:

$$
\boldsymbol{U}=\left[u_{1}, u_{2}, u_{3}, u_{4}\right]^{T}=\xi\left(\boldsymbol{X}, y_{1}, \dot{y}_{1}, \ddot{y}_{1}, y_{2}, \dot{y}_{2}, \ddot{y}_{2}, y_{3}, \dot{y}_{3}, \ddot{y}_{3}, y_{4}, \dot{y}_{4}, \ddot{y}_{4}\right)
$$

Only when the parameters of the 4-DOF PMBAMB system are invariable and accurately known, can the inversion of the 4-DOF PMBAMB system given in equation (16) be obtained. Therefore, it is difficult to acquire the satisfactory control performance by mean of the inverse system method.

The NN is one of the most commonly utilized artificial intelligence methods. Based on the theory of simulating experience-based inductive learning capacities, NNs are known as mathematical models that learn in simulation of a human brain's data-processing structure. Nowadays, NN models have been successfully achieved acceptance in a number of nonlinear engineering applications. NNs have been widely used with success in many topics, such as classification, learning, association, generalization, optimization, determining features, etc. Since the experiences of NNs are created through the information obtained from data samples, NNs can be enabled to achieve similar decisions in similar topics. In other words, the main task of an $\mathrm{NN}$ is to determine the outputs which satisfy the inputs submitted to it. In this paper, combining the ability of expressing nonlinear mapping, learning and selfadapting of NNs with the linearization and decoupling characteristic of the inverse system method, we proposed the NNI scheme. The NN is employed to approach the inversion of the 4-DOF PMBAMB system, and eight integrators are utilized to characterize the dynamic characteristics of the inverse system. Therefore, the NN and the integrators form the dynamic NN, which can realize the function of the analytical inverse system scheme, as well as achieve the static and the dynamic characteristics of the inverse model completely. 
In general, NNs are mainly composed the input layer, hidden layer and output layers. Without loss of generality, we use a three layers feed-forward NN to approach the inversion of the 4-DOF PMBAMB system. The excited function of the hidden layer is monotony slippery tangent function and the output layer is constructed by neurons with linear threshold excited function. The number of the latent neurons is a compromise between training time, output precision, and generalization capabilities. On one hand, if the number of the latent neurons is small, the $\mathrm{NN}$ will take a long time to converge or will not converge to a satisfactory error. On the other hand, with a large number of neurons, the NN will memorize the training vectors and give a large error for generalization vectors. In this paper, the structure of the $\mathrm{NN}$ is an 8-input and 8-output structure, and the number of the latent neurons is 16.

By connecting the NNI of the 4-DOF PMBAMB system with the original system, a pseudolinear system can be obtained, as illustrated in Fig. 3. The pseudolinear system, consisting of the NNI and the original system in series, is equivalent to four 2-order linear integral subsystems.

\section{Fig. 3 HERE}

\section{2-DOF Internal Model Controllers Design}

To achieve the high precision control of the 4-DOF PMBAMB system in a direct-driven spindle system, closed-loop controllers are indispensable. In this section, we will develop the design and analysis of the 2-DOF internal model controllers for the linearized plants (i.e., pseudolinear system).

Taking the pseudolinear subsystem of $A X$ - axis radial channel as an example, Fig. 4 illustrates the schematic representation of the 2-DOF internal model controller. In Fig. 4, $x_{a}^{*}$ is the reference input of $A X$ - axis radial displacement (its unit is $\mu \mathrm{m}$ ), $v$ is the control input, $x_{a}$ is the output (its unit is $\mu \mathrm{m}$ ), $d_{a x}$ is the external load including disturbance signals from external forces, and $e$ is the error which represents the effect of disturbances and plant/model mismatch if exists; $G(s)$ and $G_{a x}(s)$ are the real plant to be controlled and the predictive model of the plant(i.e., the internal model), respectively; $G_{c 1}(s)$ and $G_{c 2}(s)$ are internal model controllers, and usually known as the set point controller (in an open loop form) and the disturbance rejection controller (in a feedback structure), respectively. The most effort of the design of the internal model controller is the design of controllers $G_{c 1}(s)$ and $G_{c 2}(s)$ to obtain a reasonable tradeoff between the controller performance and robustness. Firstly, the set point controller $G_{c 1}(s)$ is chosen for good set-point response, while the disturbance rejection controller $G_{c 2}(s)$ is chosen for good disturbance rejection utilizing the inversion of the model. Secondly, low-pass filters are employed to augment $G_{c 1}(s)$ and $G_{c 2}(s)$ to provide milder action of the manipulated variables movements to improve robustness.

Fig. 4 HERE 
The transfer function of the pseudolinear subsystem of $A X$ - axis radial channel can be written as

$$
G(s)=G_{a x}(s)+G_{d}(s)=1 / s^{2}+G_{d}(s)
$$

where $G_{d}(s)$ is the arbitrary errors and uncertainties.

From Fig. 4, we can calculate the output as

$$
x_{a}(s)=\frac{x_{a}^{*}(s) G_{c 1}(s) G(s)+d_{a x}(s)\left(1-G_{c 2}(s) G_{a x}(s)\right)}{1+G_{c 2}(s) G_{d}(s)}
$$

If $G_{a x}(s)=G(s)$, i.e., the internal model is accurate (at this point $\left.G_{d}(s)=0\right)$, (18) can be rewritten as

$$
x_{a}(s)=x_{a}^{*}(s) G_{c 1}(s) G_{a x}(s)+d_{a x}(s)\left(1-G_{c 2}(s) G_{a x}(s)\right)
$$

From (19), it can be seen that the tracking characteristic is only depend on $G_{c 1}(s)$, as well as the disturbance rejection performance is only depend on $G_{c 2}(s)$, respectively. Thus, $G_{c 1}(s)$ and $G_{c 2}(s)$ can be designed as

$$
\left\{\begin{array}{l}
G_{c 1}(s)=G_{a x}^{-1}(s) Q_{1}(s) \\
G_{c 2}(s)=G_{a x}^{-1}(s) Q_{2}(s)
\end{array}\right.
$$

where $Q_{1}(s)$ and $Q_{2}(s)$ are low-pass filters, commonly chosen as

$$
\left\{\begin{array}{l}
Q_{1}(s)=\left(1+\lambda_{1} s\right)^{-2} \\
Q_{2}(s)=\left(1+\lambda_{2} s\right)^{-2}
\end{array}\right.
$$

where $\lambda_{1}$ and $\lambda_{2}$ are the tuning parameter of low-pass filters.

Furthermore, by simplifying Fig. 4, we can obtain the improved 2-DOF internal model controller as shown in Fig. 5. Here

$$
\left\{\begin{array}{l}
T(s)=\frac{G_{c 1}(s)}{G_{c 2}(s)}=\frac{\left(1+\lambda_{2} s\right)^{2}}{\left(1+\lambda_{1} s\right)^{2}} \\
G_{c}(s)=\frac{G_{c 2}(s)}{1-G_{c 2}(s) G_{a x}(s)}=\frac{s^{2}}{\left(1+\lambda_{2} s\right)^{2}-1}
\end{array}\right.
$$

Fig. 5 HERE

By selecting the proper $\lambda_{2}$, we can guarantee the stability of the closed-loop system. In addition, the larger the $\lambda_{2}$, the larger the $G_{d}(s)$ that can be acceptable. Furthermore, the smaller the $\lambda_{1}$, the better tracking performance; the smaller the $\lambda_{2}$, the better the disturbance rejection characteristic. Therefore, under the allowing model error, the effective independent control of tracking and robustness characteristics can be achieved by choosing the appropriate $\lambda_{1}$ and $\lambda_{2}$.

Similarly, we can design the 2-DOF internal model controllers for the other three $B X-, A Y$-, and $B Y$ axes radial channels in the same way. 
By introducing the 2-DOF internal model controllers into the pseudolinear subsystems, we can obtain the control block diagram of the proposed NNI-based internal model control scheme of the 4DOF PMBAMB system as shown in Fig. 6.

\section{Fig. 6 HERE}

\section{Experimental Results}

Since the 4-DOF PMBAMB system is an unstable system with the open-loop control, the close-loop controllers, i.e., PID controllers, are adopted in the data sampling process. The 4-DOF PMBAMB system is excited by random signals of the input control current components $i_{a x}, i_{b x}, i_{a y}$, and $i_{b y}$ in the $A X-$, $B X$-, $A Y$-, and $B Y$-axes in the actual operating area, and then the output signals of the primary system, i.e., the radial displacements of the rotor $x_{a}, x_{b}, y_{a}$, and $y_{b}$ in the $A X-, B X-, A Y-$, and $B Y$-axes, can be obtained. The sampling time and the sampling cycle are set $100 \mathrm{~s}$ and $0.1 \mathrm{~s}$, respectively. Therefore, the input and output signals of the original 4-DOF PMBAMB system can be sampled. From Fig. 6, we can see that besides the radial displacements of the rotor $x_{a}, x_{b}, y_{a}$, and $y_{b}$, the input signals of the $\mathrm{NN}$ also include their 1-order and 2-order derivative. Thus, the high accuracy seven-point numerical algorithm is used to calculate the 1-order and 2-order derivative of the radial displacement signals. Consequently, we can obtain the training sample sets $\left\{\ddot{x}_{a}, \dot{x}_{a}, x_{a}, \ddot{x}_{b}, \dot{x}_{b}, x_{b}, \ddot{y}_{a}, \dot{y}_{a}, y_{a}, \ddot{y}_{b}, \dot{y}_{b}, y_{b}\right\}$ and $\left\{x_{a}, x_{b}, y_{a}, y_{b}\right\}$. Furthermore, to solve the problem of slow convergence, the training sample data can be normalized into $-1 \sim+1$.

Afterwards, the aforementioned training sample sets are utilized to train the $\mathrm{NN}$, and the BP train algorithm with variable study rate is chosen to adjust the weights and thresholds of the NN. After about 500 epochs training, the training error of the $\mathrm{NN}$ is under 0.001 and met the requirement. Thus, the weights and thresholds of the NN can be saved. Therefore, the NN is successfully constructed.

To verify the proposed control scheme for the 4-DOF PMBAMB system, some experimental studies are carried out. Moreover, the results are compared with the traditional PID control method. To have a fair comparison, the inputs of the two control schemes have the same saturation limit, and they all achieved relatively good performance by adjusting the control parameters. Fig. 7 shows the photograph of the experimental test setup, and Tables I-III illustrate the system parameters as well as the parameters of the two control schemes. The proposed control algorithm is implemented in a TMS320F2812 digitalsignal-processor (DSP)-based control computer, respectively. The clock frequency of the DSP is 150 $\mathrm{MHz}$ and the PWM switching frequency is $20 \mathrm{kHz}$. Three cases were considered as follows for the 4DOF PMBAMB system.

\section{Fig. 7 HERE}




\section{TABLE I HERE \\ TABLE II HERE \\ TABLE III HERE}

\subsection{Static decoupling performance}

In this case, static decoupling control under the condition of zero speed $(\Omega=0 \mathrm{r} / \mathrm{min})$ is presented here. At $t=0.2 \mathrm{~s}$, the reference radial displacement $x_{a}$ steps from 0 to $40 \mu \mathrm{m}$, and Figs. 8 and 9 show the comparative experimental results with the traditional PID control scheme and the proposed scheme, respectively. In Figs. 8 and 9, from top to bottom are, in order, radial displacements $x_{a}, x_{b}, y_{a}$, and $y_{b}$, respectively.

\section{Fig. 8 HERE}

Fig. 9 HERE

From Fig 8, it can be seen that the sudden change of radial displacement $x_{a}$ results in a fluctuation to radial displacement $x_{b}$ with the traditional control method. On the other hand, as shown in Fig. 9, it is obvious that the sudden change of radial displacement $x_{a}$ almost has no effect on radial displacement $x_{b}$ with the proposed control scheme. In addition, to obtain a more obvious comparison, the comparative experimental result between the input and output of the radial displacement in $A X$ axis by the proposed control scheme are illustrated in Table IV. According to Fig. 9 and Table IV, a conclusion can be drawn that the proposed control scheme achieves better static decoupling performance to the traditional PID control method. Moreover, from Figs. 8 and 9, we can see that the step displacement of radial

displacement $x_{a}$ has no effect on radial displacements $y_{a}$, and $y_{b}$ whether or not the proposed control scheme has been used. This illustrates that in the case of $\Omega=0 \mathrm{r} / \mathrm{min}$, there is no coupling relationship of the radial displacements between $X$ - and $Y$-directions, which is consistent with the theory analysis we analyzed in Section II.

\subsection{Dynamic decoupling performance}

In this case, dynamic decoupling control under the condition of rotor speed $(\Omega=4000 \mathrm{r} / \mathrm{min})$ is presented. In the same way, at $t=0.2 \mathrm{~s}$, the reference radial displacement $x_{a}$ steps from 0 to $40 \mu \mathrm{m}$, and Figs. 10 and 11 illustrate the comparison with the traditional PID control scheme and the proposed one, respectively. Similarly, in Figs. 10 and 11, from top to bottom are, in order, radial displacements $x_{a}, x_{b}$, $y_{a}$, and $y_{b}$, respectively.

\section{Fig. 10 HERE}

Fig. 11 HERE

As shown in Fig. 10, with respect to the traditional control method, when the radial displacement $x_{a}$ steps from 0 to $40 \mu \mathrm{m}$ at $t=0.2 \mathrm{~s}$, there are a range of obvious fluctuations among the other three radial 
displacements $x_{b}, y_{a}$, and $y_{b}$. In other words, one radial displacement step input affects the other three radial displacement outputs. This illustrates that in the case of $\Omega=4000 \mathrm{r} / \mathrm{min}$, there is strong coupling relationship of the radial displacements between $X$ - and $Y$-directions, which is consistent with the theory analysis made in Section II.

However, as seen in Fig. 11, as for the proposed control scheme, when the radial displacement $x_{a}$ suddenly changes from 0 to $40 \mu \mathrm{m}$ at $t=0.2 \mathrm{~s}$, there are almost no fluctuations among the other three radial displacements $x_{b}, y_{a}$, and $y_{b}$. That is, one radial displacement step input almost has no effect on other three radial displacement outputs. Thus, comparing with the traditional PID control method, the proposed NNI plus internal model control scheme can achieve the decoupling control among four radial displacements $x_{a}, x_{b}, y_{a}$, and $y_{b}$, and the decoupling performance is satisfying.

\subsection{Tracking, disturbance rejection, and robustness performance}

In this case, the tracking, disturbance rejection, and robustness properties of the 4-DOF PMBAMB system under the condition of rotor speed $(\Omega=4000 \mathrm{r} / \mathrm{min})$ is verified by subjecting the 4-DOF PMBAMB system to the twisting motion, external disturbance and system parameters as well as the control parameters variation, respectively. At $t=0.2 \mathrm{~s}$, a twisting motion, with references of $40 \mu \mathrm{m},-40$ $\mu \mathrm{m},-40 \mu \mathrm{m}$, and $40 \mu \mathrm{m}$ of the radial displacements $x_{a}, x_{b}, y_{a}$, and $y_{b}$, respectively, is imposed on the 4DOF PMBAMB system of the magnetically suspended direct-driven spindle system. After $0.3 \mathrm{~s}$, a torque of $2.5 \mathrm{~N} \cdot \mathrm{m}$ is imposed on the 4-DOF PMBAMB system, and then, the current stiffness $K_{2}$ of the 4-DOF PMBAMB system varies from 280 N/A to 350 N/A at $t=0.8 \mathrm{~s}$. Since the stiffness coefficient $K_{2}$ is a function of the air gap length, bias flux and the pole face area which are constant parameters. Thus, the value of the stiffness coefficient $\mathrm{K} 2$ is also a constant (similarly, stiffness coefficient $K_{1}$ is a constant as well). In our work, in order to test the robustness (verify the variation of the system parameters) of the proposed control method, we changed the stiffness coefficient $K_{2}$ artificially in the DSP CCS software to achieve the system parameter variation with time at $\mathrm{t}=0.8 \mathrm{~s}$. Since the air gap length, bias flux and the pole face area do not change, there is no influence on the stiffness coefficient $K_{1}$. The experimental results with the traditional control scheme and the proposed one are depicted in Figs. 12 and 13, respectively. Still, in Figs. 12 and 13, from top to bottom are, in order, radial displacements $x_{a}, x_{b}, y_{a}$, and $y_{b}$, respectively.

\section{Fig. 12 HERE}

Fig. 13 HERE

\section{TABLE IV HERE}

In Fig. 12, it is obvious that as for the traditional PID control scheme, when the twisting motion takes place at $t=0.2 \mathrm{~s}$, the overshoot and the settling time are $87 \%$ and $0.17 \mathrm{~s}$, respectively. And then, when 
the torque is imposed on the 4-DOF PMBAMB system at $t=0.5 \mathrm{~s}$, the deviation values and the settling time are $28 \mu \mathrm{m}$ and $0.15 \mathrm{~s}$, respectively. In addition, when the current stiffness $K_{2}$ suddenly changes at $t$ $=0.8 \mathrm{~s}$, the deviation value and the settling time are $16 \mu \mathrm{m}$ and $0.12 \mathrm{~s}$, respectively. On the contrary, as shown in Fig. 13, regarding the proposed NNI plus internal model control scheme, when the twisting motion takes place at $t=0.2 \mathrm{~s}$, the overshoot and the settling time are $20 \%$ and $0.05 \mathrm{~s}$, respectively. When the torque is imposed on the 4-DOF PMBAMB system at $t=0.5 \mathrm{~s}$, the deviation values and the settling time are $8 \mu \mathrm{m}$ and $0.04 \mathrm{~s}$, respectively. When the current stiffness $K_{2}$ suddenly changes at $t=0.8$ $\mathrm{s}$, the deviation value and the settling time are $7 \mu \mathrm{m}$ and $0.05 \mathrm{~s}$, respectively. In addition, to obtain a more obvious comparison, the above-mentioned experimental studies between the traditional PID control method and the proposed NNI plus internal model control scheme are illustrated in Table V. According to Figs. 12, 13 and Table V, we can see that by employing the proposed control method, the tracking curves are relatively steady with small overshoots and short settling times when the twisting motion, external disturbance and parameter variation are imposed on the 4-DOF PMBAMB system, which undoubtedly verify the effectiveness of the proposed control scheme.

Based on the aforementioned results, we can come to a conclusion that the proposed NNI plus internal model control scheme can effectively improve the performance of high-precision, fast-response, and strong-robustness of the 4-DOF PMBAMB system in a magnetically suspended direct-driven spindle system.

\section{Conclusion}

To effectively improve the control precision of a 4-DOF PMBAMB system in a magnetically suspended direct-driven spindle system, a NNI plus 2-DOF internal model control scheme is proposed in this paper. The experimental results demonstrate the following: Firstly, the proposed control scheme can successfully realize the decoupling control of the 4-DOF PMBAMB system in a magnetically suspended direct-driven spindle system with fast-response and high-precision. Secondly, by introducing the 2-DOF internal model controllers, the unmodeled dynamics to the decoupling accuracy can be eliminate effectively. Moreover, the proposed control scheme is superior to the traditional decentralized PID control method in decoupling precision, tracking, disturbance rejection, and robustness properties of the controlled plant.

\section{Acknowledgments:}

This work was supported by the National Natural Science Foundation of China under Project 51305170 and 51475214, the National Science Foundation of Jiangsu Province of China under Project BK20130515 and BK20141301. 


\section{References:}

[1] J. Zhou, L. Di, C. Cheng, Y. Xu, and Z. Lin, A rotor unbalance response based approach to the identification of the closed-loop stiffness and damping coefficients of active magnetic bearings. Mechanical Systems and Signal Processing 66-67 (2016) 665-678.

[2] O. Halminen, A. Karkkainen, J. Sopanen, and A. Mikkola, Active magnetic bearing-supported rotor with misaligned cageless backup bearings: a dropdown event simulation model. Mechanical Systems and Signal Processing 50-51 (2015) 692-705.

[3] R.P. Jastrzebski, K.M. Hynynen, and A. Smirnov, H(infinity) control of active magnetic suspension. Mechanical Systems and Signal Processing 24 (2010) 995-1006.

[4] K. Jiang, C. Zhu, L. Chen, and X. Qiao, Multi-DOF rotor model based measurement of stiffness and damping for active magnetic bearing using multi-frequency excitation. Mechanical Systems and Signal Processing 60-61 (2015) 358-374.

[5] V.E. Vavilov, A.A. Gerasin, F.R. Ismagilov, and I.K. Khairullin, Stability analysis of hybrid magnetic bearings. Journal of Computer and Systems Sciences International 53 (2014) 130-136.

[6] S.M. Darbandi, M. Behzad, H. Salarieh, and H. Mehdigholi, Linear output feedback control of a threepole magnetic bearing. IEEE/ASME Transactions on Mechatronics 19 (2014) 1323-1330.

[7] T.M. Lim, D. Zhang, J. Yang, S. Cheng, S.H. Low, L.P. Chua, and X. Wu, Design and parameter estimation of hybrid magnetic bearings for blood pump applications. Mechanical Systems and Signal Processing 23 (2009) 2352-2382.

[8] N.C. Tsai, C.H. Kuo, and R.M. Lee, Regulation on radial position deviation for vertical AMB systems. Mechanical Systems and Signal Processing 21 (2007) 2777-2793.

[9] N.C. Tsai, L.W. Shih, and R.M. Lee, Counterbalance of cutting force for advanced milling operations. Mechanical Systems and Signal Processing 24 (2010) 1191-1208.

[10] S. Chen, and F. Lin, Decentralized PID neural network control for five degree-of-freedom active magnetic bearing. Engineering Applications of Artificial Intelligence 26 (2013) 962-973.

[11] N.C. Tsai, and C.W. Chiang, Spindle position regulation for wind power generators. Mechanical Systems and Signal Processing 24 (2010) 873-889.

[12] M. Lepetic, I. Skrjanc, H.G. Chiacchiarini, and D. Matko, Predictive functional control based on fuzzy model: magnetic suspension system case study. Engineering Applications of Artificial Intelligence 16 (2003) 425-430.

[13] Z. Gosiewski, and A. Mystkowski, Robust control of active magnetic suspension: Analytical and experimental results. Mechanical Systems and Signal Processing 22 (2008) 1297-1303.

[14] K. Jiang, and C. Zhu, Multi-frequency periodic vibration suppressing in active magnetic bearing-rotor systems via response matching in frequency domain. Mechanical Systems and Signal Processing 25 (2011) 1417-1429.

[15] N.C. Tsai, L.W. Shih, and R.M. Lee, Counterbalance of cutting force for advanced milling operations. Mechanical Systems and Signal Processing 24 (2010) 1191-1208.

[16] S. Folea, C.I. Muresan, R. De Keyser, and C.M. Ionescu, Theoretical analysis and experimental validation of a simplified fractional order controller for a magnetic levitation system. IEEE Transactions on Control Systems Technology 24 (2016) 756-763.

[17] S. Sivrioglu, Adaptive backstepping for switching control active magnetic bearing system with vibrating base. IET Control Theory and Applications 1 (2007) 1054-1059.

[18] L. Zhang, and K. Liu, Riccati difference equation in optimal control for magnetic bearings. Science China-Technological Sciences 55 (2012) 2107-2114.

[19] S. Park, and C. Lee, Decoupled control of a disk-type rotor equipped with a three-pole hybrid magnetic bearing. IEEE/ASME Transactions on Mechatronics 15 (2010) 793-804.

[20] A.K. Jana, Differential geometry-based adaptive nonlinear control law: application to an industrial refinery process. IEEE Transactions on Industrial Informatics 9 (2013) 2014-2022.

[21] W. Lu, C.W. Li, and C.B. Xu, Sliding mode control of a shunt hybrid active power filter based on the inverse system method. International Journal of Electrical Power and Energy Systems 57 (2014) 39-48. 
[22] X. Sun, Z. Xue, L. Chen, Z. Yang, and H. Zhu, Radial position control of a magnetically suspended rotor system in a direct-driven spindle using inverse system scheme. Transactions of the Institute of Measurement and Control online (2015) 0142331215577662.

[23] C. Cecati, J. Kolbusz, P. Rozycki, P. Siano, and B.M. Wilamowski, A novel RBF training algorithm for short-term electric load forecasting and comparative studies. IEEE Transactions on Industrial Electronics 62 (2015) 6519-6529.

[24] S. Hussain, M. Mokhtar, and J.M. Howe, Sensor failure detection, identification, and accommodation using fully connected cascade neural network. IEEE Transactions on Industrial Electronics 62 (2015) 16831692.

[25] F.D.C. Lopes, E.H. Watanabe, and L.G.B. Rolim, A control-oriented model of a PEM fuel cell stack based on NARX and NOE neural networks. IEEE Transactions on Industrial Electronics 62 (2015) 5155-5163. [26] X. Sun, L. Chen, Z. Yang, and H. Zhu, Speed-sensorless vector control of a bearingless induction motor with artificial neural network inverse speed observer. IEEE/ASME Transactions on Mechatronics 18 (2013) 1357-1366.

[27] X. Dai, D. He, X. Zhang, and T. Zhang, MIMO system invertibility and decoupling control strategies based on ANN a th-order inversion. IEE Proceedings - Control Theory and Applications 148 (2001) 125-136.

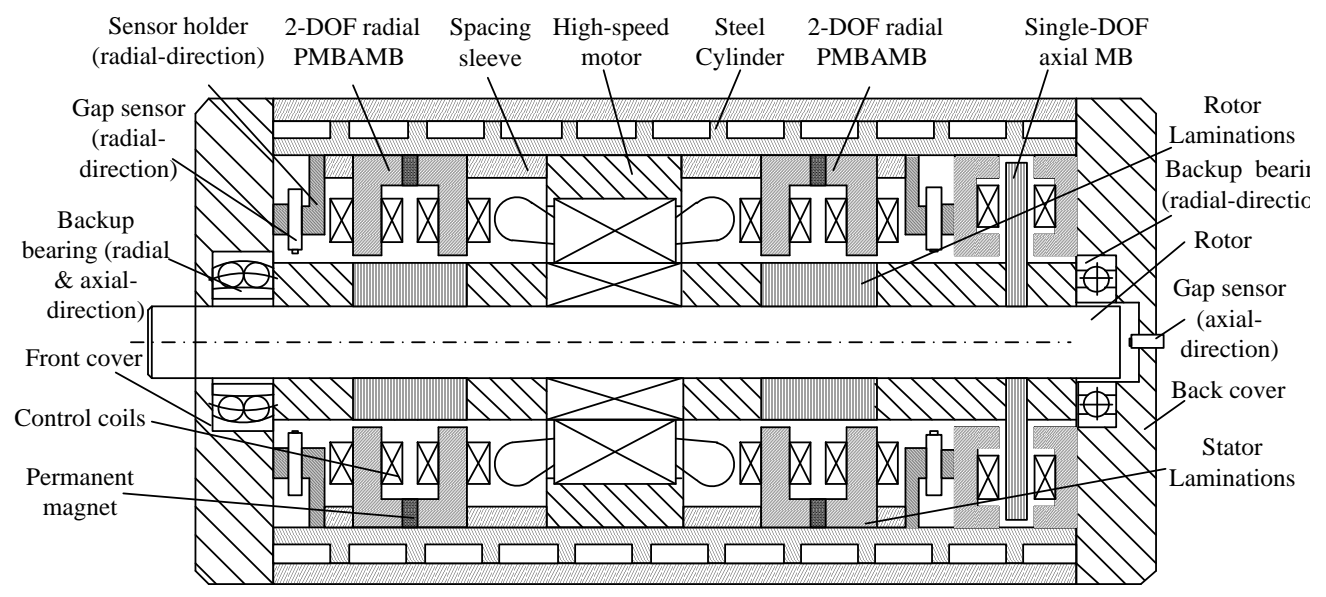

Fig. 1. Structure of a magnetically suspended direct-driven spindle system.

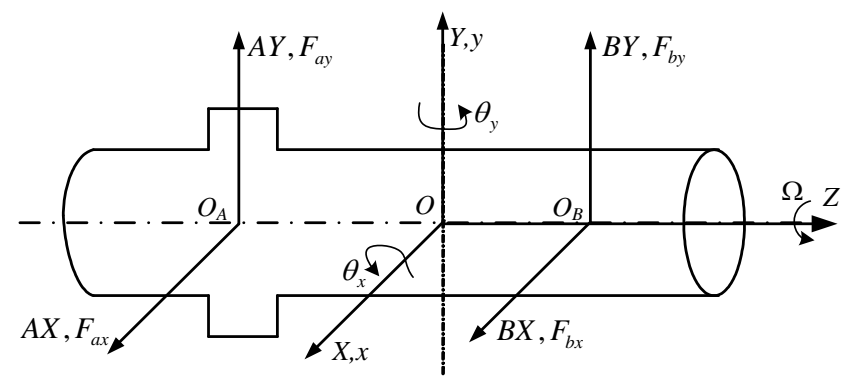

Fig. 2. Geometry relationships between the rotor and 4-DOF PMBAMB system. 

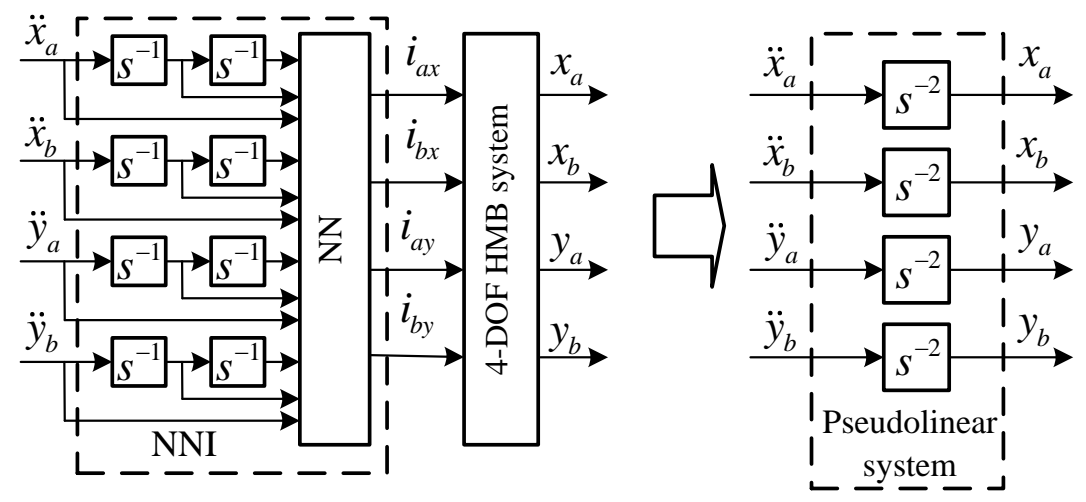

Fig. 3. Diagram of pseudolinear system.

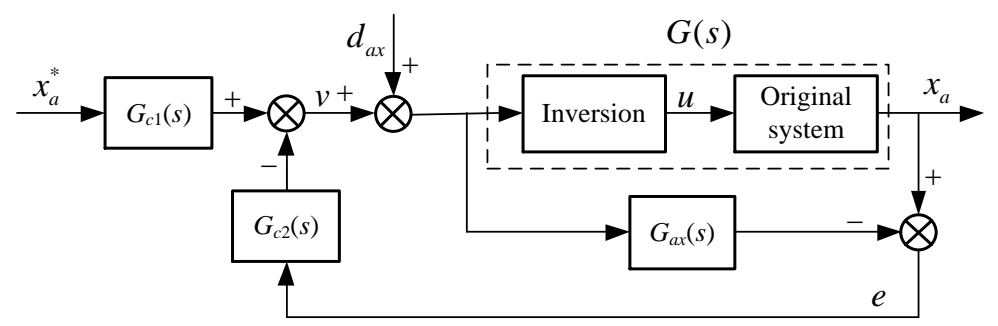

Fig. 4. The 2-DOF internal model controller structure.

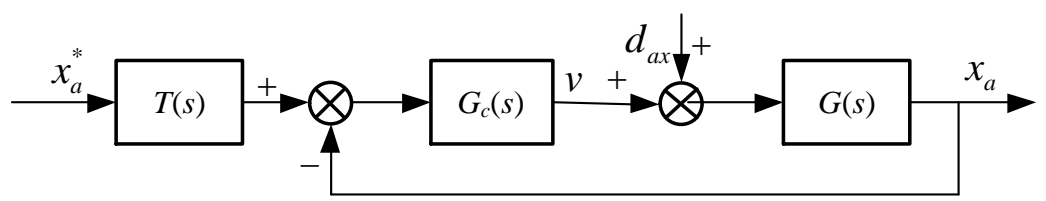

Fig. 5. The closed-loop system of the 2-DOF internal model controller.

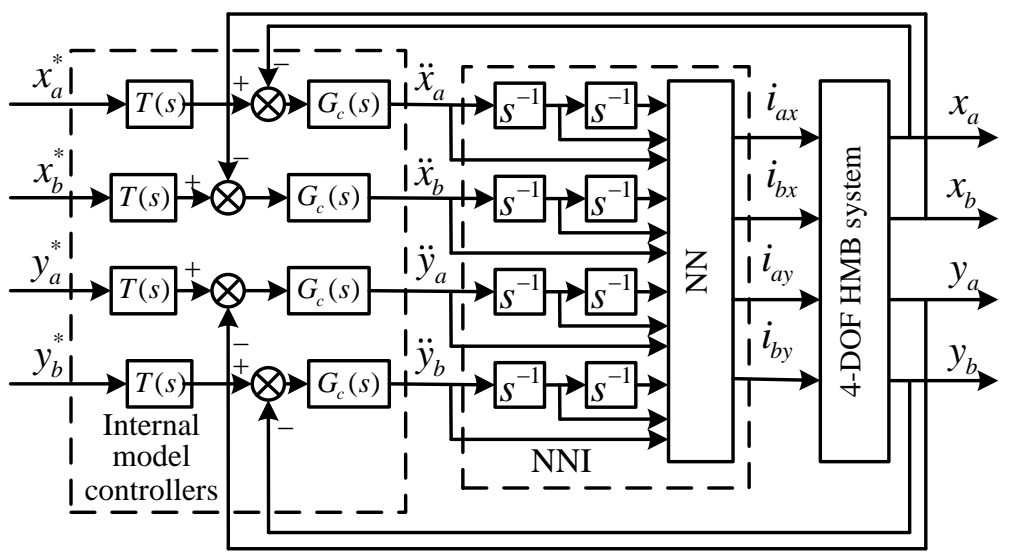

Fig. 6. Control block diagram of the entire system. 


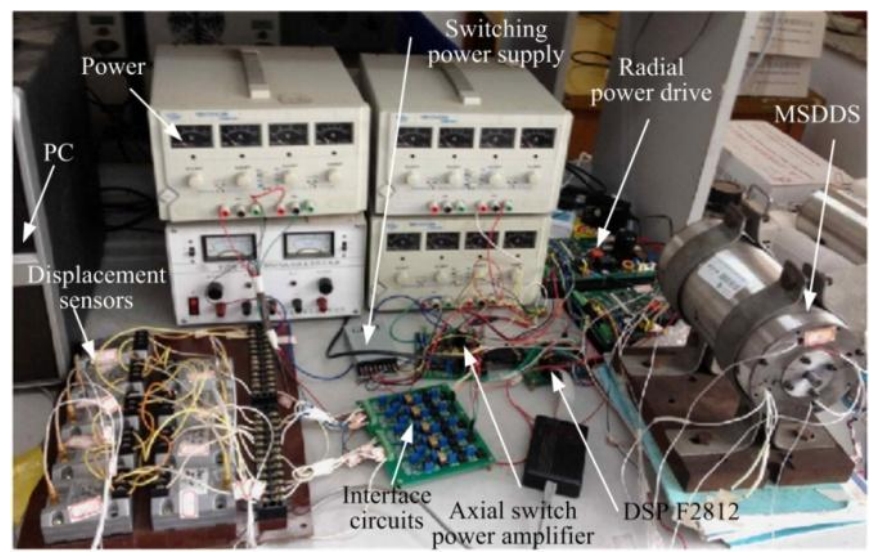

Fig. 7. Experimental test setup.

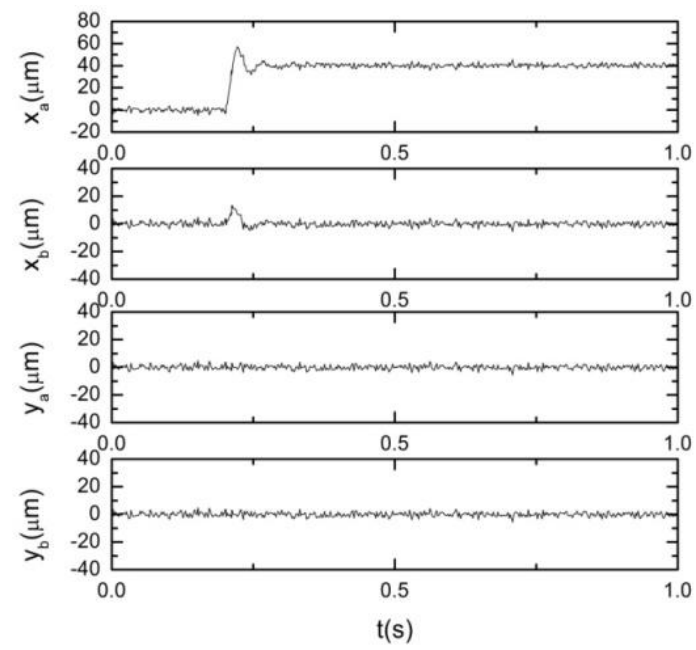

Fig. 8. Static decoupling performance with the PID control scheme under the condition $\Omega=0$.
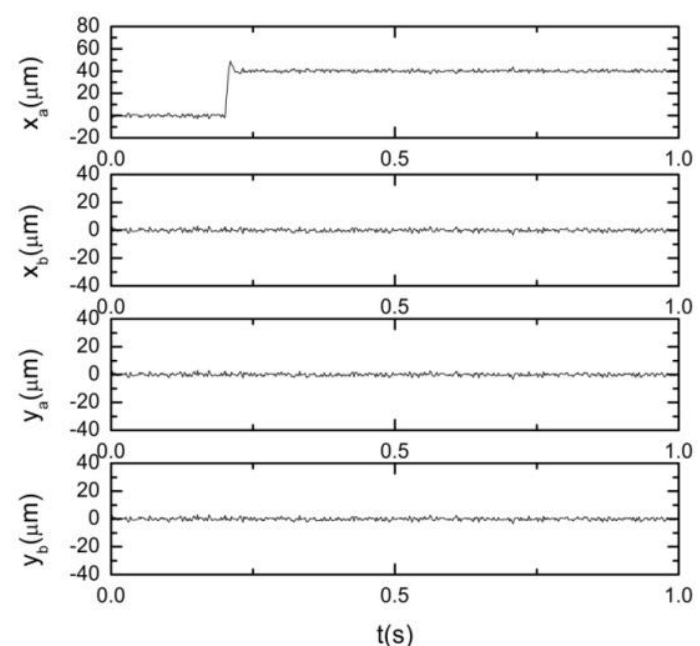

Fig. 9. Static decoupling performance with the proposed control scheme under the condition $\Omega=0$. 

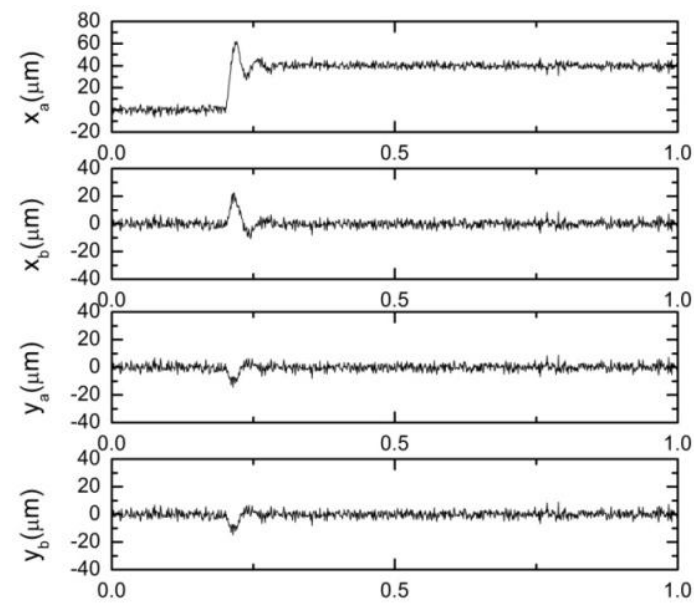

$\mathrm{t}(\mathrm{s})$

Fig. 10. Dynamic decoupling performance with the PID control scheme under the condition $\Omega=4000$ $\mathrm{r} / \mathrm{min}$.
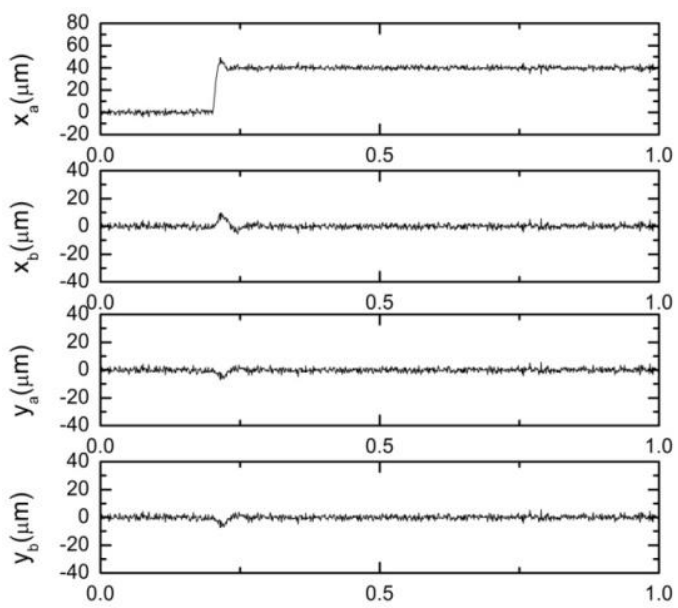

$\mathrm{t}(\mathrm{s})$

Fig. 11. Dynamic decoupling performance with the proposed control scheme under the condition $\Omega=$ $4000 \mathrm{r} / \mathrm{min}$ 


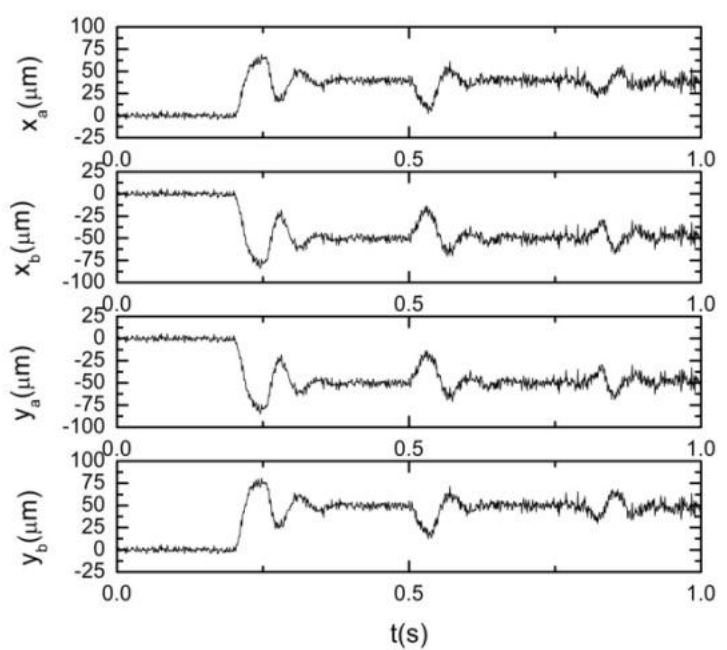

Fig. 12. Tracking, disturbance rejection, and robustness performance with the PID control scheme under the condition $\Omega=4000 \mathrm{r} / \mathrm{min}$.

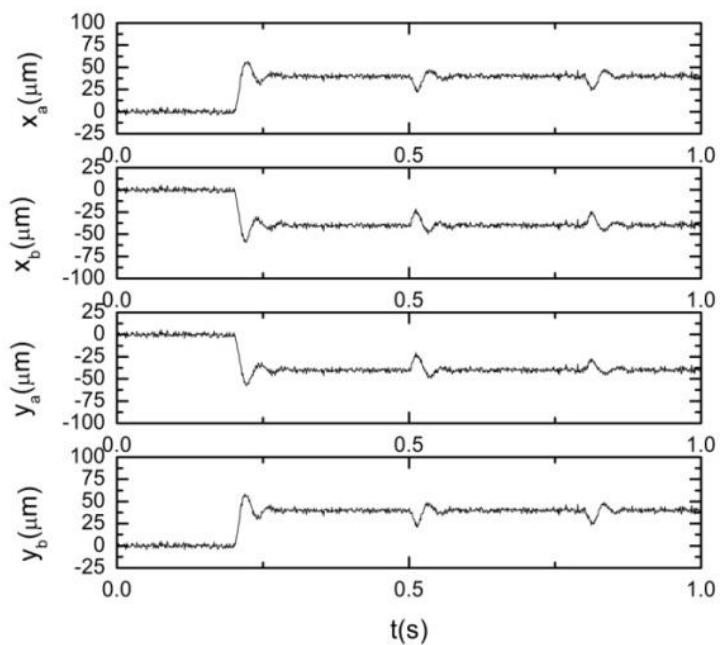

Fig. 13. Tracking, disturbance rejection, and robustness performance with the proposed control scheme under the condition $\Omega=4000 \mathrm{r} / \mathrm{min}$. 
TABLE I

Parameters of 4-DOF PMBAMB system

\begin{tabular}{cccc}
\hline Parameter & Value & Parameter & Value \\
\hline Moments of inertia $J_{x}=J_{y}$ & $0.0018 \mathrm{~kg} \cdot \mathrm{m}^{2}$ & Mass of the rotor $m$ & $1.0 \mathrm{~kg}$ \\
Moments of inertia $J_{z}$ & $0.0034 \mathrm{~kg} \cdot \mathrm{m}^{2}$ & $\begin{array}{c}\text { Nominal radial } \\
\text { airgap length } \delta_{0}\end{array}$ & $500 \mu \mathrm{m}$ \\
Displacement stiffness & $7.6 \times 10^{5} \mathrm{~N} / \mathrm{m}$ & Distance $l_{a}$ & $0.0731 \mathrm{~m}$ \\
coefficient $K_{1}$ & & Distance $l_{b}$ & $0.0627 \mathrm{~m}$ \\
Current stiffness coefficient $K_{2}$ & $320 \mathrm{~N} / \mathrm{A}$ &
\end{tabular}

TABLE II

Parameters of the proposed scheme

\begin{tabular}{cccc}
\hline Parameter & Value & Parameter & Value \\
\hline$\lambda_{1}$ & 0.015 & $\lambda_{2}$ & 0.01 \\
\hline
\end{tabular}

TABLE III

Parameters of the traditional PID scheme

\begin{tabular}{cccc}
\hline Parameter & Value & Parameter & Value \\
\hline$K_{p}$ & 35 & $K_{i}$ & 13 \\
$K_{d}$ & 0.1 & & \\
\hline
\end{tabular}

TABLE IV

Comparative result between the input and output of the radial displacement in $A X$ axis

\begin{tabular}{|c|c|c|}
\hline \multirow{2}{*}{} & \multicolumn{2}{|c|}{$A X$ axis } \\
\cline { 2 - 3 } & Input & output \\
\hline Settling time & $0 \mathrm{~s}$ & $0.04 \mathrm{~s}$ \\
\hline Overshoot & 0 & $18 \%$ \\
\hline
\end{tabular}




\section{TABLE V}

Comparative results between two control schemes

\begin{tabular}{|c|c|c|c|}
\hline \multicolumn{2}{|c|}{} & $\begin{array}{c}\text { Traditional } \\
\text { scheme }\end{array}$ & $\begin{array}{c}\text { Proposed } \\
\text { scheme }\end{array}$ \\
\hline \multirow{2}{*}{ Tracking } & Settling time & $0.17 \mathrm{~s}$ & $0.05 \mathrm{~s}$ \\
\cline { 2 - 4 } & Overshoot & $87 \%$ & $20 \%$ \\
\hline \multirow{2}{*}{$\begin{array}{c}\text { Disturbance } \\
\text { rejection }\end{array}$} & Settling time & $0.25 \mathrm{~s}$ & $0.04 \mathrm{~s}$ \\
\cline { 2 - 4 } Robustness & Deviation value & $28 \mu \mathrm{m}$ & $8 \mu \mathrm{m}$ \\
\cline { 2 - 4 } & Settling time & $0.12 \mathrm{~s}$ & $0.05 \mathrm{~s}$ \\
\cline { 2 - 4 } & Deviation value & $16 \mu \mathrm{m}$ & $7 \mu \mathrm{m}$ \\
\hline
\end{tabular}

